\title{
EXISTENCE AND STABILITY OF PERIODIC SOLUTIONS \\ FOR A CLASS OF GENERALIZED NONAUTONOMOUS \\ NEURAL NETWORKS WITH DISTRIBUTED DELAYS
}

YONGKUN LI, LIFEI ZHU, AND WENXIANG LIU

Received 22 April 2004 and in revised form 28 February 2005

By using the continuation theorem of coincidence degree theory and Lyapunov functions, we study the existence and global stability of periodic solutions for a class of generalized nonautonomous neural networks with distributed delays.

\section{Introduction}

The study of the dynamics of neural networks has greatly attracted the attention of the scientific community because of their promising potential for the tasks of classification, associative memory, and parallel computations, and their ability to solve difficult optimization problems. Many papers $[1,4,5,8,9,10,12,13,14,15]$ have been devoted to discussing the stability of neural networks with delays. Recently, the authors of [7] have studied the globally exponential stability of the trivial solution for the following generalized neural networks with distributed delays

$$
\begin{aligned}
\dot{x}_{i}(t)= & -d_{i}\left(x_{i}(t)\right)+\sum_{j=1}^{n} \omega_{i j}\left(x_{1}(t), \ldots, x_{n}(t)\right) f_{j}\left(x_{j}(t)\right)+\sum_{j=1}^{n} \omega_{i j}^{\tau}\left(x_{1}(t), \ldots, x_{n}(t)\right) \\
& \times \int_{-\infty}^{t} k_{i j}(t-s) f_{j}\left(x_{j}(s)\right) d s, \quad i=1,2, \ldots, n,
\end{aligned}
$$

where $x_{i}$ is the state of the $i$-neuron at time $t, A=\left(\omega_{i j}\right)$ and $B=\left(\omega_{i j}^{\tau}\right)$ are $n \times n$ interconnection matrices, respectively, $f_{j}$ is an activation function. However, under some practical circumstances, the connection weights, the activation functions, and the rate functions of most neural network models (i.e., $\omega_{i j}, \omega_{i j}^{\tau}, f_{j}$, and $d_{i}$ in system (1.1)) depend not only on the state $x_{i}(t)$ but also on the time $t$, so the nonautonomous system can be applied in wider fields. In this paper, we are concerned with the following nonautonomous neural network system

$$
\begin{aligned}
\dot{x}_{i}(t)= & -d_{i}\left(t, x_{i}(t)\right)+\sum_{j=1}^{n} \omega_{i j}\left(t, x_{1}(t), \ldots, x_{n}(t)\right) f_{j}\left(t, x_{j}(t)\right)+\sum_{j=1}^{n} \omega_{i j}^{\tau}\left(t, x_{1}(t), \ldots, x_{n}(t)\right) \\
& \times \int_{-\infty}^{t} k_{i j}(t-s) f_{j}\left(t, x_{j}(s)\right) d s, \quad i=1,2, \ldots, n .
\end{aligned}
$$


It is well known that studies on neural network dynamical systems not only involve a discussion of stability properties, but also involve many dynamic behaviors such as periodic oscillatory behavior, almost-periodic oscillatory properties, chaos, and bifurcation [11], and to the best of our knowledge, few authors considered the existence of periodic solutions for the model (1.2). Our purpose of this paper is to prove the existence and stability of periodic solutions of (1.2).

Throughout this paper, we assume that

(H1) for each $i=1,2, \ldots, n, d_{i} \in C\left(R^{2}, R\right)$ is $T$-periodic with respect to its first argument, and $\lim _{u \rightarrow+\infty} d_{i}(t, u)=+\infty$ and $\lim _{u \rightarrow-\infty} d_{i}(t, u)=-\infty$ are uniformly in $t$, respectively;

(H2) for each $i, j=1,2, \ldots, n, k_{i j}$ is real-valued nonnegative continuous function defined on $[0, \infty)$ and $\int_{0}^{\infty} k_{i j}(s) d s=1$;

(H3) for each $i, j=1,2, \ldots, n, f_{i} \in C\left(R^{2}, R\right), \omega_{i j}^{\tau}, \omega_{i j} \in C\left(R^{n+1}, R\right)$ are bounded and $f_{i}$, $\omega_{i j}^{\tau}$, and $\omega_{i j}$ are $T$-periodic with respect to their first arguments, respectively.

The organization of this paper is as follows. In the second section, we prove the existence of periodic solutions of system (1.2) by applying the continuation theorem of coincidence degree theory. In the third section, some sufficient conditions are obtained to show the global asymptotic stability of periodic solutions of system (1.2).

\section{Existence of positive periodic solutions}

In this section, based on the Mawhin's continuation theorem, we will study the existence of at least one positive periodic solution of (1.2). First, we will make some preparations.

Let $X, Y$ be normed vector spaces, $L:$ Dom $L \subset X \rightarrow Y$ a linear mapping, and $N: X \rightarrow$ $Y$ a continuous mapping. The mapping $L$ will be called a Fredholm mapping of index zero if $\operatorname{dim} \operatorname{Ker} L=\operatorname{codim} \operatorname{Im} L<+\infty$ and $\operatorname{Im} L$ is closed in $Y$. If $L$ is a Fredholm mapping of index zero and there exist continuous projectors $P: X \rightarrow X$ and $Q: Y \rightarrow Y$ such that $\operatorname{Im} P=$ $\operatorname{Ker} L, \operatorname{Ker} Q=\operatorname{Im} L=\operatorname{Im}(I-Q)$, it follows that mapping $\left.L\right|_{\operatorname{Dom} L \cap \operatorname{Ker} P}:(I-P) X \rightarrow \operatorname{Im} L$ is invertible. We denote the inverse of that mapping by $K_{P}$. If $\Omega$ is an open bounded subset of $X$, the mapping $N$ will be called $L$-compact on $\bar{\Omega}$ if $Q N(\bar{\Omega})$ is bounded and $K_{P}(I-Q) N: \bar{\Omega} \rightarrow X$ is compact. Since $\operatorname{Im} Q$ is isomorphic to $\operatorname{Ker} L$, there exists an isomorphism $J: \operatorname{Im} Q \rightarrow \operatorname{Ker} L$.

Now, we introduce Mawhin's continuation theorem [2, page 40] as follows.

Lemma 2.1. Let $\Omega \subset X$ be an open bounded set and let $N: X \rightarrow Y$ be a continuous operator which is L-compact on $\bar{\Omega}$. Assume

(a) for each $\lambda \in(0,1), x \in \partial \Omega \cap \operatorname{Dom} L, L x \neq \lambda N x$;

(b) for each $x \in \partial \Omega \cap \operatorname{Ker} L, Q N x \neq 0$;

(c) $\operatorname{deg}(J N Q, \Omega \cap \operatorname{Ker} L, 0) \neq 0$.

Then $L x=N x$ has at least one solution in $\bar{\Omega} \cap \operatorname{Dom} L$.

Theorem 2.2. Assume that (H1)-(H3) hold. Then the system (1.2) has at least one Tperiodic solution.

Proof. In order to apply the continuation theorem of coincidence degree theory to establish the existence of a $T$-periodic solution of (1.2), we take

$$
X=Y=\left\{x \in C\left(R, R^{n}\right): x(t+T)=x(t), t \in R\right\}
$$


and denote

$$
\|x\|=\sup _{t \in[0, T]} \sum_{i=1}^{n}\left|x_{i}(t)\right|,
$$

then $X$ is a Banach space. Set

$$
L: \operatorname{Dom} L \cap X, \quad L x=\dot{x}(t), \quad x \in X,
$$

where $\operatorname{Dom} L=\left\{x \in C^{1}\left(R, R^{n}\right)\right\}$ and $N: X \rightarrow X$,

$$
\begin{aligned}
\left(N x_{i}\right)(t)= & -d_{i}\left(t, x_{i}(t)\right)+\sum_{j=1}^{n} \omega_{i j}\left(t, x_{1}(t), \ldots, x_{n}(t)\right) f_{j}\left(t, x_{j}(t)\right)+\sum_{j=1}^{n} \omega_{i j}^{\tau}\left(t, x_{1}(t), \ldots, x_{n}(t)\right) \\
& \times \int_{-\infty}^{t} k_{i j}(t-s) f_{j}\left(t, x_{j}(s)\right) d s, \quad i=1,2, \ldots, n .
\end{aligned}
$$

Define two projectors $P$ and $Q$ as

$$
Q x=P x=\frac{1}{T} \int_{0}^{T} x(s) d s, \quad x \in X .
$$

Clearly, $\operatorname{Ker} L=R^{n}, \operatorname{Im} L=\left\{\left(x_{1}, \ldots, x_{n}\right)^{T} \in X: \int_{0}^{T} x_{i}(t) d t=0, i=1,2, \ldots, n\right\}$ is closed in $X$ and $\operatorname{dim} \operatorname{Ker} L=\operatorname{codim} \operatorname{Im} L=n$. Hence, $L$ is a Fredholm mapping of index 0 . Furthermore, similar to the proof of [6, Theorem 1$]$, one can easily show that $N$ is $L$-compact on $\bar{\Omega}$ with any open bounded set $\Omega \subset X$.

Corresponding to operator equation $L x=\lambda N x, \lambda \in(0,1)$, we have

$$
\begin{aligned}
\frac{d x_{i}}{d t}= & -\lambda d_{i}\left(t, x_{i}(t)\right)+\lambda \sum_{j=1}^{n} \omega_{i j}\left(t, x_{1}(t), \ldots, x_{n}(t)\right) f_{j}\left(t, x_{j}(t)\right)+\lambda \sum_{j=1}^{n} \omega_{i j}^{\tau}\left(t, x_{1}(t), \ldots, x_{n}(t)\right) \\
& \times \int_{-\infty}^{t} k_{i j}(t-s) f_{j}\left(t, x_{j}(s)\right) d s, \quad i=1,2, \ldots, n .
\end{aligned}
$$

Suppose that $x=\left(x_{1}, \ldots, x_{n}\right) \in X$ is a solution of (2.6) for some $\lambda \in(0,1)$. Let $\xi_{i} \in[0, T]$ such that $x_{i}\left(\xi_{i}\right)=\max _{t \in[0, \omega]} x_{i}(t), i=1,2, \ldots, n$, then

$$
\begin{aligned}
& -\lambda d_{i}\left(\xi_{i}, x_{i}\left(\xi_{i}\right)\right)+\lambda \sum_{j=1}^{n} \omega_{i j}\left(\xi_{i}, x_{1}\left(\xi_{i}\right), \ldots, x_{n}\left(\xi_{i}\right)\right) f_{j}\left(\xi_{i}, x_{j}\left(\xi_{i}\right)\right) \\
& \quad+\lambda \sum_{j=1}^{n} \omega_{i j}^{\tau}\left(\xi_{i}, x_{1}\left(\xi_{i}\right), \ldots, x_{n}\left(\xi_{i}\right)\right) \int_{-\infty}^{\xi_{i}} k_{i j}\left(\xi_{i}-s\right) f_{j}\left(\xi_{i}, x_{j}(s)\right) d s=0, \quad i=1,2, \ldots, n .
\end{aligned}
$$


1000 Periodic solutions for nonautonomous neural networks

In view of (H2) and (H3), we have

$$
\begin{aligned}
d_{i}\left(\xi_{i}, x_{i}\left(\xi_{i}\right)\right) \leq & \sum_{j=1}^{n}\left|\omega_{i j}\left(\xi_{i}, x_{1}\left(\xi_{i}\right), \ldots, x_{n}\left(\xi_{i}\right)\right)\right|\left|f_{j}\left(\xi_{i}, x_{j}\left(\xi_{i}\right)\right)\right| \\
& +\sum_{j=1}^{n}\left|\omega_{i j}^{\tau}\left(\xi_{i}, x_{1}\left(\xi_{i}\right), \ldots, x_{n}\left(\xi_{i}\right)\right)\right| \\
& \times\left|\int_{-\infty}^{\xi_{i}} k_{i j}\left(\xi_{i}-s\right) f_{j}\left(\xi_{i}, x_{j}(s)\right) d s\right| \\
\leq & n \bar{\omega} \bar{f}+n \overline{\omega^{\tau}} \bar{f}, \quad i=1,2, \ldots, n
\end{aligned}
$$

where $\bar{\omega}=\max \left\{\left|\omega_{i j}\left(t, \nu_{1}, \ldots, v_{n}\right)\right|,\left(t, v_{1}, \ldots, v_{n}\right)^{T} \in R^{n+1}, i, j=1,2, \ldots, n\right\}, \overline{\omega^{\tau}}=\max \left\{\mid \omega_{i j}^{\tau}(t\right.$, $\left.\left.v_{1}, \ldots, v_{n}\right) \mid,\left(t, v_{1}, \ldots, v_{n}\right)^{T} \in R^{n+1}, i, j=1,2, \ldots, n\right\}, \bar{f}=\max \left\{\left|f_{j}\left(t, \mu_{j}\right)\right|,\left(t, \mu_{j}\right)^{T} \in R^{2}, j=\right.$ $1,2, \ldots, n\}$. According to (H1) and (2.8), we know that there exists a constant $A_{1}>0$ such that

$$
x_{i}\left(\xi_{i}\right) \leq A_{1}, \quad i=1,2, \ldots, n
$$

Similarly, let $\eta_{i} \in[0, \omega]$ such that $x_{i}\left(\eta_{i}\right)=\min _{t \in[0, \omega]} x_{i}(t), i=1,2, \ldots, n$, then

$$
\begin{aligned}
& -\lambda d_{i}\left(\eta_{i}, x_{i}\left(\eta_{i}\right)\right)+\lambda \sum_{j=1}^{n} \omega_{i j}\left(\eta_{i}, x_{1}\left(\eta_{i}\right), \ldots, x_{n}\left(\eta_{i}\right)\right) f_{j}\left(\eta_{i}, x_{j}\left(\eta_{i}\right)\right) \\
& \quad+\lambda \sum_{j=1}^{n} \omega_{i j}^{\tau}\left(\eta_{i}, x_{1}\left(\eta_{i}\right), \ldots, x_{n}\left(\eta_{i}\right)\right) \int_{-\infty}^{\eta_{i}} k_{i j}\left(\eta_{i}-s\right) f_{j}\left(\eta_{i}, x_{j}(s)\right) d s=0, \quad i=1,2, \ldots, n .
\end{aligned}
$$

Then,

$$
\begin{aligned}
d_{i}\left(\eta_{i}, x_{i}\left(\eta_{i}\right)\right) \geq & -\sum_{j=1}^{n}\left|\omega_{i j}\left(\eta_{i}, x_{1}\left(\eta_{i}\right), \ldots, x_{n}\left(\eta_{i}\right)\right)\right|\left|f_{j}\left(\eta_{i}, x_{j}\left(\eta_{i}\right)\right)\right| \\
& -\sum_{j=1}^{n}\left|\omega_{i j}^{\tau}\left(\eta_{i}, x_{1}\left(\eta_{i}\right), \ldots, x_{n}\left(\eta_{i}\right)\right)\right| \\
& \times\left|\int_{-\infty}^{\eta_{i}} k_{i j}\left(\eta_{i}-s\right) f_{j}\left(\eta_{i}, x_{j}(s)\right) d s\right| \\
\geq & -n \bar{\omega} \bar{f}-n \overline{\omega^{\tau}} \bar{f}, \quad i=1,2, \ldots, n,
\end{aligned}
$$

where $\bar{\omega}, \bar{f}, \overline{\omega^{\tau}}$ is the same as those in (2.8). Therefore, there exists a constant $A_{2}>0$ such that

$$
x_{i}\left(\eta_{i}\right) \geq-A_{2}, \quad i=1,2, \ldots, n
$$


Denote $D=\max \left\{n A_{1}, n A_{2}\right\}+E$, where $E$ is a positive constant, clearly, $D$ is independent of $\lambda$. Now, we take $\Omega=\{x \in X,\|x\|<D\}$. This $\Omega$ satisfies condition (a) in Lemma 2.1.

When $x \in \partial \Omega \cap \operatorname{Ker} L=\partial \Omega \cap R^{n}, x$ is a constant vector in $R^{n}$ with $\|x\|=D$. Then

$$
\begin{aligned}
x^{T} Q N x=-\frac{1}{T} \sum_{i=1}^{n} x_{i} \int_{0}^{T}\left[d_{i}\left(t, x_{i}\right)-\sum_{j=1}^{n} \omega_{i j}\left(t, x_{1}, \ldots, x_{n}\right) f_{j}\left(t, x_{j}\right)\right. \\
\left.\quad-\sum_{j=1}^{n} \omega_{i j}^{\tau}\left(t, x_{1}, \ldots, x_{n}\right) \int_{-\infty}^{t} k_{i j}(t-s) f_{j}\left(t, x_{j}\right) d s\right] d t \\
\leq-\frac{1}{T} \sum_{i=1}^{n} x_{i} \int_{0}^{T}\left[d_{i}\left(t, x_{i}\right)-n \bar{\omega} \bar{f}-n \overline{\omega^{\tau}} \bar{f}\right] d t \\
<0, \quad i=1,2, \ldots, n .
\end{aligned}
$$

If necessary, we can let $E$ be greater such that $-(1 / T) \sum_{i=1}^{n} x_{i} \int_{0}^{T}\left[d_{i}\left(t, x_{i}\right)-n \bar{\omega} \bar{f}-n \overline{\omega^{\tau}} \bar{f}\right] d t$ $<0$. This prove that condition (b) in Lemma 2.1 is satisfied.

Finally, we will prove that condition (c) in Lemma 2.1 is also satisfied. Let $\psi(\nu ; x)=$ $-v x+(1-\nu) Q N x$, then for any $x \in \partial \Omega \cap \operatorname{Ker} L, x^{T} \psi(\nu, x)<0$, we get

$$
\operatorname{deg}(J Q M, \Omega \cap \operatorname{Ker} L, 0) \neq 0 .
$$

Thus, by Lemma 2.1, we conclude that $L x=N x$ has at least one solution in $X$, that is, (1.2) has at least one positive $T$-periodic solution. The proof is complete.

\section{Global asymptotic stability of periodic solutions}

Let $x(t)=\left(x_{1}(t), \ldots, x_{n}(t)\right)$ be any solution of $(1.2)$ and $x^{*}(t)=\left(x_{1}^{*}(t), \ldots, x_{n}^{*}(t)\right)$ a $T$ periodic solution of (1.2). Set $u(t)=x(t)-x^{*}(t)$, then

$$
\frac{d u_{i}(t)}{d t}=-\alpha_{i}\left(u_{i}(t)\right)+\beta_{i}\left(u_{i}(t)\right)+\gamma_{i}\left(u_{i}(t)\right), \quad i=1,2, \ldots, n,
$$

where

$$
\begin{aligned}
\alpha_{i}\left(u_{i}(t)\right)= & d_{i}\left(t, x_{i}(t)\right)-d_{i}\left(t, x_{i}^{*}(t)\right), \\
\beta_{i}\left(u_{i}(t)\right)= & \sum_{j=1}^{n} \omega_{i j}\left(t, x_{1}(t), \ldots, x_{n}(t)\right) f_{j}\left(t, x_{j}(t)\right)-\sum_{j=1}^{n} \omega_{i j}\left(t, x_{1}^{*}(t), \ldots, x_{n}^{*}(t)\right) f_{j}\left(t, x_{j}^{*}(t)\right), \\
\gamma_{i}\left(u_{i}(t)\right)= & \sum_{j=1}^{n} \omega_{i j}^{\tau}\left(t, x_{1}(t), \ldots, x_{n}(t)\right) \int_{-\infty}^{t} k_{i j}(t-s) f_{j}\left(t, x_{j}(s)\right) d s \\
& -\sum_{j=1}^{n} \omega_{i j}^{\tau}\left(t, x_{1}^{*}(t), \ldots, x_{n}^{*}(t)\right) \int_{-\infty}^{t} k_{i j}(t-s) f_{j}\left(t, x_{j}^{*}(s)\right) d s .
\end{aligned}
$$


1002 Periodic solutions for nonautonomous neural networks

In the sequel, we will use the following notations:

$$
\bar{\omega}_{i j}=\max \left\{\omega_{i j}\left(t, v_{1}, \ldots, v_{n}\right),\left(t, v_{1}, \ldots, v_{n}\right)^{T} \in R^{n+1}\right\}, \quad \bar{f}_{j}=\max \left\{f_{j}(t, \mu),(t, \mu)^{T} \in R^{2}\right\} .
$$

Theorem 3.1. Assume that (H1)-(H3) hold. Furthermore, assume that

(H4) for each $i=1,2, \ldots, n, f_{i}: R^{2} \rightarrow R$ is globally Lipschitz continuous with a Lipschitz constant $F_{i}$ with respect to its second argument,

(H5) for each $i=1,2, \ldots, n, d_{i} \in C^{1}\left(R^{2}, R\right)$ and there exists a constant $D_{i} \geq 0$ such that

$$
\left[d_{i}(t, u)\right]_{u}^{\prime} \geq D_{i}, \quad u \in R
$$

(H6) for each $i, j=1,2, \ldots, n$, there exist constants ${ }^{l} B_{i j} \geq 0$ and ${ }^{l} B_{i j}^{\tau} \geq 0$ such that

$$
\begin{aligned}
& \left|\omega_{i j}\left(t, x_{1}(t), \ldots, x_{n}(t)\right)-\omega_{i j}\left(t, y_{1}(t), \ldots, y_{n}(t)\right)\right| \leq \sum_{l=1}^{n}{ }^{l} B_{i j}\left|x_{l}(t)-y_{l}(t)\right|, \quad t \in R, \\
& \left|\omega_{i j}^{\tau}\left(t, x_{1}(t), \ldots, x_{n}(t)\right)-\omega_{i j}^{\tau}\left(t, y_{1}(t), \ldots, y_{n}(t)\right)\right| \leq \sum_{l=1}^{n}{ }^{l} B_{i j}^{\tau}\left|x_{l}(t)-y_{l}(t)\right|, \quad t \in R,
\end{aligned}
$$

(H7) for each $i=1,2, \ldots, n$,

$$
M_{i}:=D_{i}-F_{i} \sum_{j=1}^{n}\left(\bar{\omega}_{j i}+\bar{\omega}_{j i}^{\tau}\right)-\sum_{j=1}^{n} \sum_{l=1}^{n}\left({ }^{i} B_{l j}+{ }^{i} B_{l j}^{\tau}\right) \bar{f}_{j}>0,
$$

then (1.2) has a unique T-periodic solution which is globally asymptotically stable.

Proof. We consider the Lyapunov function

$$
V(t)=V_{1}(t)+V_{2}(t)
$$

where

$$
\begin{aligned}
& V_{1}(t)=\sum_{i=1}^{n}\left|u_{i}(t)\right| \\
& V_{2}(t)=\sum_{i=1}^{n} \sum_{j=1}^{n} \bar{\omega}_{i j}^{\tau} F_{j} \int_{0}^{\infty} K_{i j}(s) \int_{t-s}^{t}\left|u_{j}(z)\right| d z d s .
\end{aligned}
$$


Calculating the derivatives of $V_{1}$ and $V_{2}$ along the solution of (3.1), respectively,

$$
\begin{aligned}
& \left.\frac{d u}{d t}\right|_{(3.1)}=\sum_{i=1}^{n} \operatorname{sign}\left(u_{i}(t)\right) \dot{u}_{i}(t) \\
& =\sum_{i=1}^{n} \operatorname{sign}\left(u_{i}(t)\right)\left\{-\alpha_{i}\left(u_{i}(t)\right)+\beta_{i}\left(u_{i}(t)\right)+\gamma_{i}\left(u_{i}(t)\right)\right\} \\
& \leq \sum_{i=1}^{n}\left\{-D_{i}\left|u_{i}(t)\right|+\left|\beta_{i}\left(u_{i}(t)\right)\right|+\left|\gamma_{i}\left(u_{i}(t)\right)\right|\right\} \\
& \leq \sum_{i=1}^{n}\left\{-D_{i}\left|u_{i}(t)\right|+\sum_{j=1}^{n}\left|\omega_{i j}\left(t, x_{1}(t), \ldots, x_{n}(t)\right)\right|\left|f_{j}\left(t, x_{j}(t)\right)-f_{j}\left(t, x_{j}^{*}(t)\right)\right|\right. \\
& +\sum_{j=1}^{n}\left|\omega_{i j}\left(t, x_{1}(t), \ldots, x_{n}(t)\right)-\omega_{i j}\left(t, x_{1}^{*}(t), \ldots, x_{n}^{*}(t)\right)\right|\left|f_{j}\left(t, x_{j}^{*}(t)\right)\right| \\
& +\sum_{j=1}^{n}\left|\omega_{i j}^{\tau}\left(t, x_{1}(t), \ldots, x_{n}(t)\right)\right| \int_{-\infty}^{t} k_{i j}(t-s) \\
& \times\left|f_{j}\left(t, x_{j}(s)\right)-f_{j}\left(t, x_{j}^{*}(s)\right)\right| d s \\
& +\sum_{j=1}^{n} \mid \omega_{i j}^{\tau}\left(t, x_{1}(t), \ldots, x_{n}(t)\right) \\
& \left.-\omega_{i j}^{\tau}\left(t, x_{1}^{*}(t), \ldots, x_{n}^{*}(t)\right)|| \int_{-\infty}^{t} k_{i j}(t-s) f_{j}\left(t, x_{j}^{*}(s)\right) d s \mid\right\}, \\
& \leq \sum_{i=1}^{n}\left\{-D_{i}\left|u_{i}(t)\right|+\sum_{j=1}^{n} \bar{\omega}_{i j} F_{j}\left|u_{j}(t)\right|+\sum_{j=1}^{n} \sum_{l=1}^{n}{ }^{l} B_{i j}\left|u_{l}(t)\right| \bar{f}_{j}\right. \\
& \left.+\sum_{j=1}^{n} \bar{\omega}_{i j}^{\tau} \int_{-\infty}^{t} k_{i j}(t-s) F_{j}\left|u_{j}(s)\right| d s+\sum_{j=1}^{n} \sum_{l=1}^{n}{ }^{l} B_{i j}^{\tau}\left|u_{l}(t)\right| \bar{f}_{j}\right\} \\
& =\sum_{i=1}^{n}\left\{-D_{i}\left|u_{i}(t)\right|+\sum_{j=1}^{n} \bar{\omega}_{i j} F_{j}\left|u_{j}(t)\right|+\sum_{j=1}^{n} \sum_{l=1}^{n}{ }^{l} B_{i j}\left|u_{l}(t)\right| \bar{f}_{j}\right. \\
& \left.+\sum_{j=1}^{n} \bar{\omega}_{i j}^{\tau} F_{j} \int_{0}^{\infty} k_{i j}(s)\left|u_{j}(t-s)\right| d s+\sum_{j=1}^{n} \sum_{l=1}^{n}{ }^{l} B_{i j}^{\tau}\left|u_{l}(t)\right| \bar{f}_{j}\right\}, \\
& \left.\frac{d V_{2}}{d t}\right|_{(3.1)}=\sum_{i=1}^{n}\left(\sum_{j=1}^{n} \bar{\omega}_{i j}^{\tau} F_{j} \int_{0}^{\infty} k_{i j}(s)\left|u_{j}(t)\right| d s-\sum_{j=1}^{n} \bar{\omega}_{i j}^{\tau} F_{j} \int_{0}^{\infty} k_{i j}(s)\left|u_{j}(t-s)\right| d s\right) \text {. }
\end{aligned}
$$

So,

$$
\begin{aligned}
\left.\frac{d V(t)}{d t}\right|_{(3.1)} \leq \sum_{i=1}^{n}\{ & -D_{i}\left|u_{i}(t)\right|+\sum_{j=1}^{n} \bar{\omega}_{i j} F_{j}\left|u_{j}(t)\right|+\sum_{j=1}^{n} \sum_{l=1}^{n}\left({ }^{l} B_{i j}+{ }^{l} B_{i j}^{\tau}\right)\left|u_{l}(t)\right| \bar{f}_{j} \\
& \left.+\sum_{j=1}^{n} \bar{\omega}_{i j}^{\tau} F_{j}\left|u_{j}(t)\right|\right\}
\end{aligned}
$$


1004 Periodic solutions for nonautonomous neural networks

$$
\begin{aligned}
= & \sum_{i=1}^{n}\left\{-D_{i}\left|u_{i}(t)\right|+\sum_{j=1}^{n}\left(\bar{\omega}_{i j}+\bar{\omega}_{i j}^{\tau}\right) F_{j}\left|u_{j}(t)\right|\right. \\
& \left.+\sum_{j=1}^{n} \sum_{l=1}^{n}\left({ }^{l} B_{i j}+{ }^{l} B_{i j}^{\tau}\right)\left|u_{l}(t)\right| \bar{f}_{j}\right\} \\
\leq & \sum_{i=1}^{n}\left\{-D_{i}+F_{i} \sum_{j=1}^{n}\left(\bar{\omega}_{j i}+\bar{\omega}_{j i}^{\tau}\right)+\sum_{j=1}^{n} \sum_{l=1}^{n}\left({ }^{i} B_{l j}+{ }^{i} B_{l j}^{\tau}\right) \bar{f}_{j}\right\}\left|u_{i}(t)\right| \\
\leq & 0 .
\end{aligned}
$$

In view of (3.7) and (3.10), we see that $\sum_{i=1}^{n}\left|u_{i}(t)\right|$ is bounded for all $t \geq 0$. For the proof of Theorem 2.2, it follows that for each $i=1,2, \ldots, n, x_{i}^{*}(t)$ is bounded. Hence the solutions of (1.2) exist and are bounded for all $t \geq 0$. Integrating both sides of (3.11) from 0 to $t$, we get

$$
V(t)+\int_{0}^{t} \sum_{i=1}^{n} M_{i}\left|u_{i}(s)\right| d s \leq V(0),
$$

which implies $u_{i}(t) \in L^{1}[0, \infty)$. Therefore, by Barbalatt's lemma [3, Lemma 1.2.2, page 4], we have

$$
\lim _{t \rightarrow \infty} u_{i}(t)=0
$$

This completes the proof.

\section{Acknowledgments}

This work is supported by the National Natural Sciences Foundation of China under Grant 10361006 and the Natural Sciences Foundation of Yunnan Province under Grant 2003A0001M.

\section{References}

[1] M. Dong, Global exponential stability and existence of periodic solutions of CNNs with delays, Phys. Lett. A 300 (2002), no. 1, 49-57.

[2] R. E. Gaines and J. L. Mawhin, Coincidence Degree, and Nonlinear Differential Equations, Springer, Berlin, 1977.

[3] K. Gopalsamy, Stability and Oscillations in Delay Differential Equations of Population Dynamics, Mathematics and Its Applications, vol. 74, Kluwer Academic Publishers, Dordrecht, 1992.

[4] K. Gopalsamy and X. Z. He, Stability in asymmetric Hopfield nets with transmission delays, Phys. D 76 (1994), no. 4, 344-358.

[5] M. Joy, On the global convergence of a class of functional-differential equations with applications in neural network theory, J. Math. Anal. Appl. 232 (1999), no. 1, 61-81.

[6] Y. Li and Y. Kuang, Periodic solutions of periodic delay Lotka-Volterra equations and systems, J. Math. Anal. Appl. 255 (2001), no. 1, 260-280. 
[7] X. Liao, K. W. Wong, and C. Li, Global exponential stability for a class of generalized neural networks with distributed delays, Nonlinear Anal. Real World Appl. 5 (2004), no. 3, 527547.

[8] T. Roska, C. W. Wu, and L. O. Chua, Stability of cellular neural networks with dominant nonlinear and delay-type templates, IEEE Trans. Circuits Systems I Fund. Theory Appl. 40 (1993), no. 4, 270-272.

[9] V. Sree Hari Rao and B. R. M. Phaneendra, Global dynamics of bidirectional associative memory neural networks involving transmission delays and dead zones, Neural Networks 12 (1999), $455-465$.

[10] P. van den Driessche and X. Zou, Global attractivity in delayed Hopfield neural network models, SIAM J. Appl. Math. 58 (1998), no. 6, 1878-1890.

[11] J. Wei and S. Ruan, Stability and bifurcation in a neural network model with two delays, Phys. D 130 (1999), no. 3-4, 255-272.

[12] Y. Yi, P. A. Heng, and K. S. Leung, Convergence analysis of cellular neural networks with unbounded delay, IEEE Trans. Circuits Systems I Fund. Theory Appl. 48 (2001), no. 6, 680687.

[13] J. Zhang, Absolutely exponential stability in delayed cellular neural networks, Int. J. Circuit Theory and Applications 30 (2002), no. 4, 395-409.

[14] Global stability analysis in delayed cellular neural networks, Comput. Math. Appl. 45 (2003), no. 10-11, 1707-1720.

[15] - Globally exponential stability of neural networks with variable delays, IEEE Trans. Circuits Systems I Fund. Theory Appl. 50 (2003), no. 2, 288-291.

Yongkun Li: Department of Mathematics, Yunnan University, Kunming, Yunnan 650091, China E-mail address: yklie@ynu.edu.cn

Lifei Zhu: Department of Mathematics and Statistics, McGill University, Montreal, QC, Canada H3A 2K6

E-mail address: zhu@math.mcgill.ca

Wenxiang Liu: Department of Mathematical and Statistical Sciences, University of Alberta, Edmonton, AB, Canada T6G 2G1

E-mail address: wliu@math.ualberta.ca 


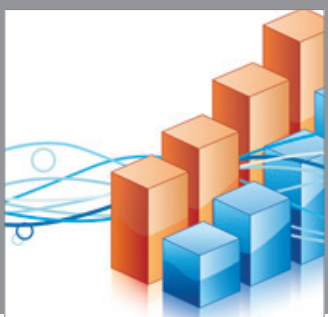

Advances in

Operations Research

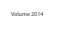

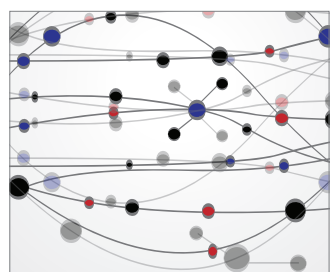

\section{The Scientific} World Journal
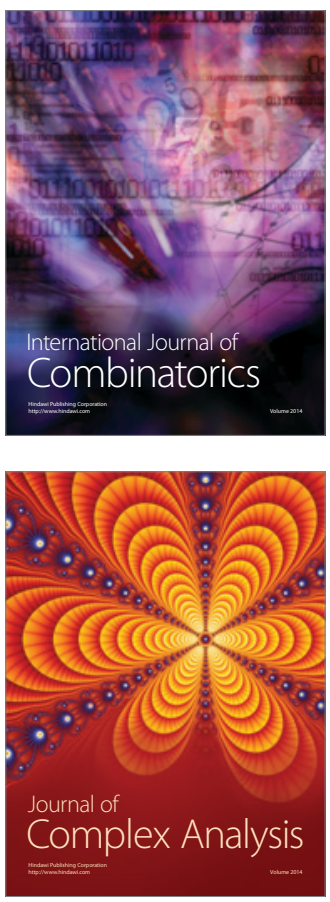

International Journal of

Mathematics and

Mathematical

Sciences
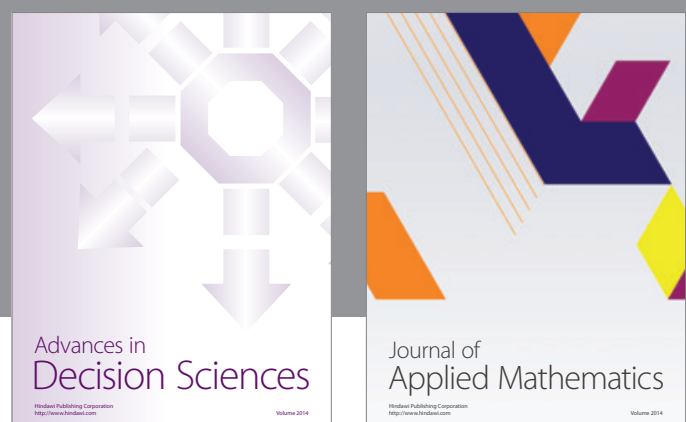

Journal of

Applied Mathematics
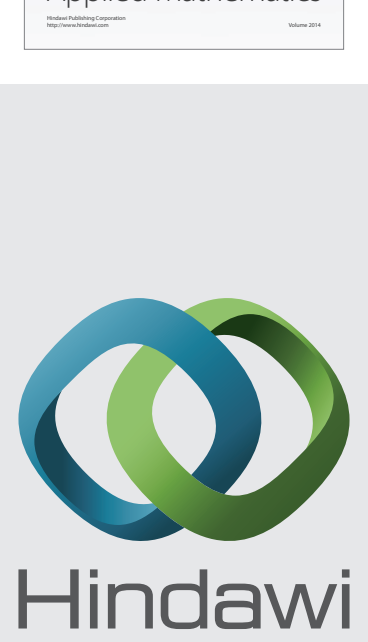

Submit your manuscripts at http://www.hindawi.com
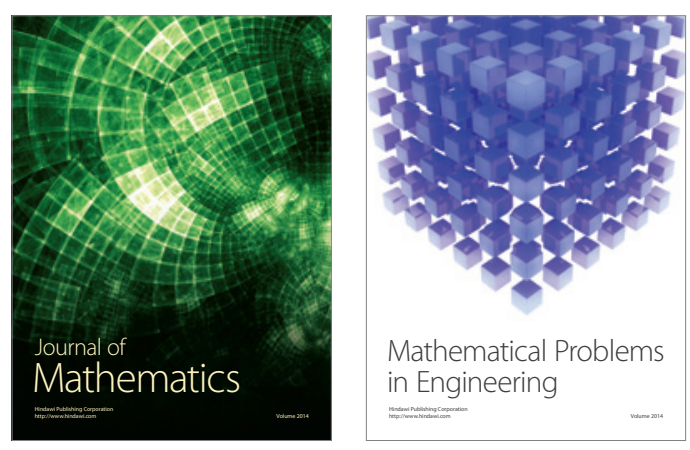

Mathematical Problems in Engineering
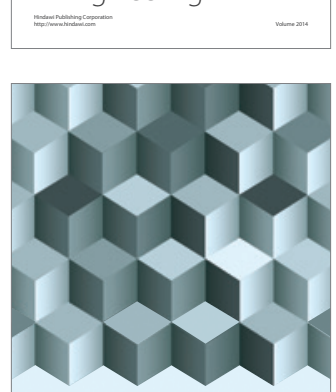

Journal of

Function Spaces
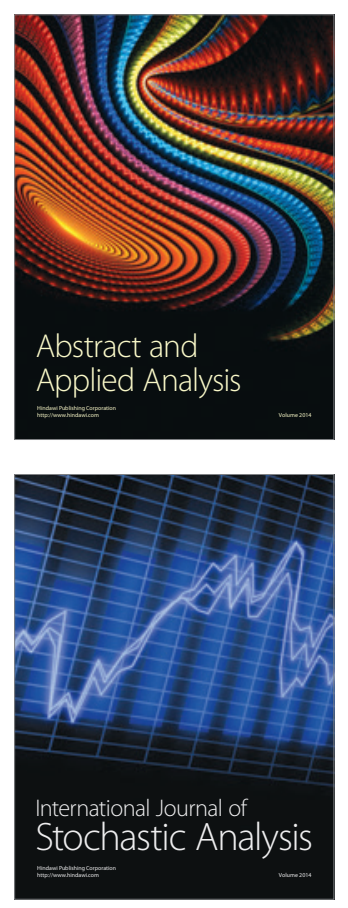

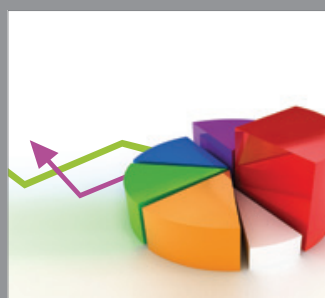

ournal of

Probability and Statistics

Promensencen
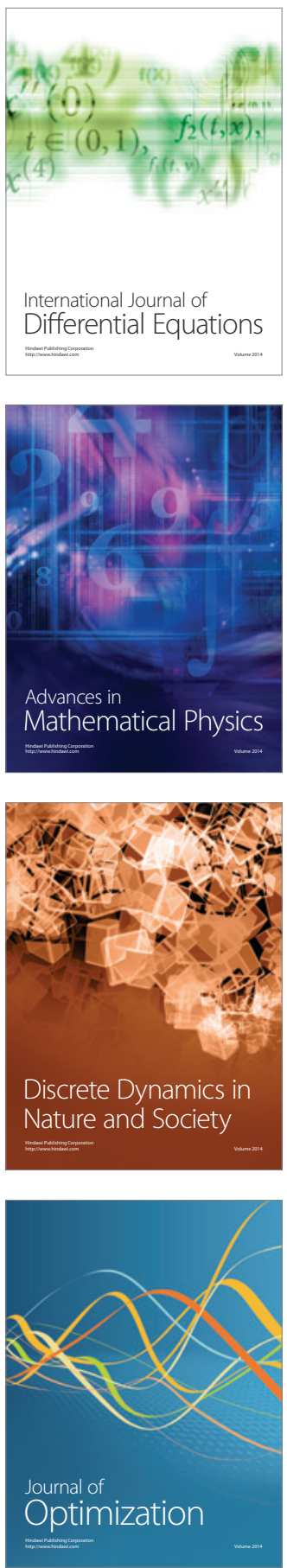